\title{
FERRAMENTAS PARA INSPEÇÃO E ANÁLISE DE REDES WIRELESSHART: COMPARAÇÃO E AVALIAÇÃO DOS MÉTODOS EXISTENTES E PROPOSTA DE UMA NOVA FERRAMENTA
}

\author{
Tatiane M. Machado ${ }^{1}$ Ivan MulleR $^{1}$, Jean M. Winter ${ }^{1}$, Victor H. Dickow ${ }^{1}$, João C. NetTo ${ }^{2}$, Carlos E. \\ PEREIRA ${ }^{1}$.
}
1. Departamento de Engenharia Elétrica, Universidade Federal do Rio Grande do Sul
E-mails: $\{$ tatiane.machado, ivan.muller, jean.winter, vic-
tor.dickow\} eufrgs.br, cpereiralece.ufrgs.br

\section{Departamento de Informática, Universidade Federal do Rio Grande do Sul \\ E-mail: nettodinfo.ufrgs.br}

\begin{abstract}
The WirelessHART protocol has been increasingly applied in the monitoring and control of industrial processes due to its high reliability and robustness. However, because it is a recent protocol, there are still research to be developed, being one of the areas related to the inspection and monitoring of WirelessHART networks in real environments. This paper presents a comparative analysis of tools for analyzing WirelessHART networks currently available in the literature and proposes a new tool, which has the advantages of mobility and inspection on all 15 channels of the WirelessHART network using only one radio, which is set dynamically during network operation. It is a solution of hardware and software that provides analysis of the logical and physical characteristics of the network, having the capability of measuring the energy level in channels concurrently with logical aspects of the links.
\end{abstract}

Keywords— WirelessHART, Analysis of wireless networks, IEEE 802.15.4, Systems automation.

\begin{abstract}
Resumo- O protocolo WirelessHART tem sido cada vez mais aplicado no monitoramento e controle de processos industriais, devido à sua alta confiabilidade e robustez. No entanto, por se tratar de um protocolo recente, ainda há pesquisas a serem desenvolvidas, sendo uma das áreas referente à inspeção e monitoramento de redes WirelessHART em ambientes reais. Este trabalho apresenta uma análise comparativa das ferramentas para análise de redes WirelessHART disponíveis atualmente na literatura e propõe uma nova ferramenta, que apresenta como vantagens a mobilidade e a inspeção em todos os 15 canais da rede WirelessHART utilizando apenas um rádio, que é programado dinamicamente durante o funcionamento da rede. Trata-se de uma solução de hardware e software que proporciona análise lógica e física da rede, possuindo a capacidade de medição do nível de energia nos canais, concomitantemente com aspectos lógicos dos enlaces.
\end{abstract}

Palavras-chave— WirelessHART, Análise de redes sem fio, IEEE 802.15.4, Sistemas de automação.

\section{Introdução}

O controle e monitoramento de processos no ambiente industrial vêm sofrendo mudanças nos últimos anos, devido ao crescimento e avanço das pesquisas relacionadas a redes sem fio para indústria. As redes sem fio apresentam muitas vantagens sobre as redes cabeadas, entre elas: (i) grande redução ou ausência de custo para instalação dos cabos; (ii) os nós da rede podem ser colocados em qualquer ponto da planta, onde muitas vezes não seria possível a colocação de cabos; (iii) baixo custo de manutenção, uma vez que não existe o custo associado à movimentação e troca de cabos; (iv) a rede pode ser aumentada sob demanda, sem necessidade de alteração da infraestrutura pré-existente. Entretanto, o ambiente industrial exige alta confiabilidade, ou seja, é necessário haver uma perfeita comunicação entre os dispositivos da rede, os requisitos temporais devem ser cumpridos e a integridade e segurança das mensagens que trafegam pela rede devem ser garantidas. Todas estas características devem ser atendidas sob as condições críticas do ambiente industrial, tais como: temperaturas elevadas, umidade, ruídos eletromagnéticos e obstáculos físicos.
Muitos protocolos industriais para comunicação sem fio têm sido propostos, como por exemplo: ZigBee PRO, ISA SP 100.11a, WIA-PA e WirelessHART (Muller, 2011). Em paralelo, surge a necessidade de ferramentas que permitam monitorar, avaliar, inspecionar e diagnosticar propriedades de desempenho e integridade dos sistemas de comunicação sem fio. Este trabalho objetiva o estudo de ferramentas para inspeção e análise de redes WirelessHART (WH). É imprescindível que os desenvolvedores de dispositivos $\mathrm{WH}$ e os usuários destas redes possuam ferramentas que permitam uma análise aprofundada sobre a dinâmica da rede.

O trabalho está organizado da seguinte forma: na seção 2 são discutidas as ferramentas existentes atualmente na literatura. Na seção 3, é proposta uma nova ferramenta para análise de redes $\mathrm{WH}$ e na seção 4 e 5 são apresentados os resultados e conclusões, respectivamente.

\section{Ferramentas para inspeção e análise de redes WirelessHART}

Nesta seção, serão apresentadas as ferramentas para inspeção e análise de redes WH existentes atualmente na literatura. 


\subsection{Porta de manutenção}

Todos os dispositivos de campo WH devem possuir uma porta de manutenção, que é utilizada para provisionamento e manutenção da rede. Antes que um dispositivo de campo possa se unir à rede, é necessário que duas informações sejam configuradas localmente: Join Key e Network ID. O Network ID identifica a rede em que o dispositivo pretende se unir e a Join Key é uma chave para encriptação das mensagens iniciais entre o dispositivo e o gerenciador de rede (HCF, 2009).

Em Lima (2012), é proposta uma ferramenta para análise local da rede WH através da porta de manutenção, sendo esta não apenas utilizada para fazer o comissionamento do dispositivo, mas também para fazer uma análise local da rede, através de comunicações do tipo requisição/resposta HART. A configuração proposta é ilustrada na Figura 1. Um computador é conectado ao dispositivo de campo através da porta de manutenção RS485 e os seguintes comandos são executados:

- Ler/escrever modo burst (comandos 103, 104, 105, 109);

- Ler lista de vizinhos (comando 780);

- Ler apelido do dispositivo (comando 781);

- Ler lista de superframes (comando 783) e lista de links (comando 784).

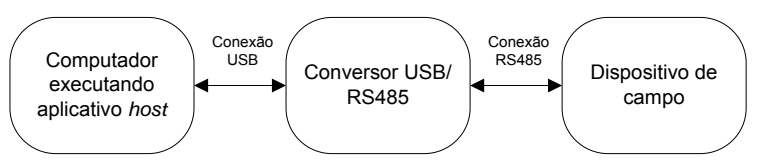

Figura 1. Configuração proposta por Lima (2012)

O aplicativo host possibilita que o usuário envie os comandos anteriormente citados para o dispositivo de campo e obtenha as respostas. A porta de manutenção é capaz de comunicar-se diretamente com o dispositivo sem sobrecarregar a rede. Desta forma, os dados são obtidos mais rapidamente do que através de consultas ao gerenciador de rede através do gateway. Com os comandos implementados, é possível obter informações importantes a respeito do funcionamento da rede. A leitura da lista de links, por exemplo, possibilita determinar com quais vizinhos o dispositivo está se comunicando e em qual intervalo de tempo. Também é possível deduzir a topologia da rede estabelecida pelo gerenciador da rede.

Esta ferramenta possui grande aplicação em laboratórios para estudo do protocolo WH e também durante o desenvolvimento de dispositivos de campo. Porém, é necessário modificar a pilha do protocolo, para que o dispositivo possa responder a comandos que seriam normalmente enviados pelo gerenciador de rede.

\subsection{HART-sobre-UDP}

O gerenciador de rede contém todas as informações necessárias para o funcionamento da rede $\mathrm{WH}$, como por exemplo: lista completa das rotas, conexões e dispositivos de rede. Tais informações são muito úteis para que se possa fazer um estudo e uma análise aprofundada a respeito da operação das redes WH.

Winter (2011) propôs uma ferramenta capaz de obter informações relacionadas ao comportamento da rede WH e aos status dos dispositivos. Isto inclui, por exemplo, a análise dos dispositivos que estão na rede, topologia da rede e links entre os dispositivos. Foi desenvolvida uma aplicação, que é executada em um computador, e que utiliza o protocolo HARTsobre-UDP para se comunicar com o gerenciador de rede WH. A aplicação é baseada em comandos HART e WH, que são utilizados para obter os dados desejados a respeito da rede. Os comandos são encapsulados no padrão UDP e enviados através do gateway aos dispositivos de campo, que respondem às requisições. Para avaliar o sistema proposto, foi realizado um estudo de caso em que uma rede WH é formada por cinco dispositivos com distanciamentos e obstáculos previamente definidos. A aplicação proposta se conecta ao gerenciador de rede através do gateway, obtendo e armazenando diversos dados associados aos comandos durante algumas horas. Com os dados armazenados, é possível estudar a dinâmica da rede e chegar a conclusões a respeito de diversos parâmetros e comportamentos.

\subsection{Ferramentas baseadas em sniffers}

$\mathrm{Na}$ literatura, existem diversas propostas de ferramentas para análise de redes WH baseadas em sniffers (dispositivos passivos para captura de dados da rede). As redes WH utilizam a camada física definida pelo padrão IEEE 802.15.4, operando na frequência de 2,4 GHz. A camada de enlace utiliza a técnica TDMA para arbitração do meio, com saltos de canais a cada slot de tempo. Embora o padrão IEEE 802.15.4 na faixa de $2,4 \mathrm{GHz}$ defina 16 canais, o protocolo WH utiliza apenas 15 canais, uma vez que o último canal (canal 26) não é disponível internacionalmente de forma restrita. Então, é necessário que o sniffer de rede esteja apto a capturar dados nos 15 canais definidos pela norma, caso contrário, não será adequado para inspeção de redes WH.

O monitoramento da rede através do uso de sniffers apresenta a vantagem de se tratar de uma inspeção passiva, ou seja, que não interfere no funcionamento da rede. Entretanto, a obtenção dos dados da rede está relacionada à área de cobertura do sniffer, que é da mesma ordem da área de cobertura de um dispositivo de rede. A seguir, são descritas as ferramentas para análise de redes WH baseadas em sniffers, existentes atualmente na literatura. 


\section{A. Wi-Analys}

Todos os dispositivos WH devem ser completamente testados e registrados pela HART Communication Foundation (HCF), a fim de garantir a compatibilidade com o protocolo e o cumprimento dos requisitos temporais exigidos pela norma. Para isso, a $\mathrm{HCF}$ desenvolveu especificações de testes detalhadas e ferramentas de teste, as quais são explicadas no trabalho de Han (2009).

Foram propostas três ferramentas, que juntas fornecem um ambiente de verificação de compatibilidade completo para dispositivos WH:

- Wi-HTest: tem como objetivo automatizar a execução dos testes definidos pela $\mathrm{HCF}$ para redes WH. É capaz de construir os pacotes de teste em tempo real e enviá-los ao dispositivo em teste através de um rádio IEEE 802.15.4. Os testes podem ser divididos em duas categorias: etapa de união do dispositivo à rede (joining) e comunicação normal de dados. No processo de joining, o Wi-HTest realiza uma sequência de troca de mensagens com o dispositivo em teste e verifica se o dispositivo se uniu à rede corretamente. Já no caso de comunicação normal de dados, o Wi-HTest transmite pacotes de dados corretos ou insere erros nos dados e verifica o comportamento do dispositivo nestas condições.

- Wi-Analys: é um sniffer para capturar pacotes de redes WH em tempo real. O equipamento foi projetado para capturar todas as transmissões IEEE 802.15.4 operando na faixa de frequência de 2,4 $\mathrm{GHz}$, mas filtrando as transmissões a partir de dispositivos WH. O receptor tem a capacidade de capturar dados nos 16 canais do padrão IEEE 802.15.4 simultaneamente e com uma velocidade de até 1000 mensagens por segundo. O Wi-Analys é composto por dezesseis receptores de rádio, um concentrador de dados e um software executando em uma estação de trabalho, o qual armazena todas as mensagens capturadas. As mensagens são mostradas ao usuário através de um formulário online ou offline, através de um arquivo de $\log$ de mensagens anteriormente capturadas. As mensagens são interpretadas e os dados, da camada física até a camada de aplicação, podem ser mostrados em colunas separadamente. Além disso, as mensagens encriptadas são decifradas e mostradas ao usuário como um texto. Para tanto, o sniffer deve ter a join key correta previamente programada. O Wi-Analys é um produto standalone da HCF para ser usado como uma ferramenta de monitoramento da rede WH em tempo real ou em conjunto com os equipamentos certificadores.

- Post process suite: este conjunto de equipamentos analisa os arquivos de $\log$ gerados pelo $\mathrm{Wi}$ Analys durante a execução dos casos de teste pelo Wi-HTest para verificar se o dispositivo seguiu a norma rigorosamente durante o teste, especialmente no que diz respeito aos requisitos temporais.

Das ferramentas propostas pela HCF no trabalho de Han (2009), o sniffer Wi-Analys é uma opção para monitoramento de redes WH. No entanto, apresenta a desvantagem de necessitar de uma estação de trabalho conectada ao sniffer para a coleta de dados. Em redes reais, isto pode ser inviável dependendo do local onde se deseja instalar o sniffer. Ainda assim a ferramenta apresenta grande utilidade para uso em laboratórios durante o estudo do protocolo $\mathrm{WH}$, especialmente durante o desenvolvimento da pilha do protocolo.

\section{B. Analisador de rede distribuído}

Depari (2009) propôs um analisador de rede WH distribuído. Uma vez que um sniffer tem uma área de cobertura limitada, ou seja, pode não abranger todos os dispositivos da rede, foi proposto um instrumento distribuído, baseado no uso de diversos sniffers interconectados a uma estação de monitoramento. Os dados coletados pelos diversos sniffers são enviados para uma rede de medição e encaminhados à estação de monitoramento, a qual pode trocar informações com o gerenciador de rede e pode executar uma aplicação para visualizar os pacotes capturados. Uma análise detalhada dos dados pode ser feita offline por meio de um software desenvolvido pelo usuário. Neste trabalho, a rede de medição utilizada pelos autores é uma rede Ethernet, ou seja, cabeada. Este fato se opõe às vantagens oferecidas pelas redes sem fio, tais como: escalabilidade e flexibilidade, uma vez que o uso da ferramenta fica restrito a locais passíveis de cobertura por cabos, inviabilizando sua utilização em campo. Os autores também sugerem que a rede Ethernet pode ser substituída por uma rede sem fio, o que eliminaria a necessidade de cabos. No entanto, o uso de uma rede sem fio para transmissão dos dados dos sniffers até a estação de monitoramento pode gerar problemas de coexistência de redes, devendo ser analisado com cautela.

\section{Analisador de 16 canais}

Krätzig (2009) propôs um analisador de pacotes para redes sem fio IEEE 802.15.4, independente das camadas superiores do protocolo. A ferramenta pode capturar dados em 16 canais em paralelo a uma taxa de amostragem de até 3200 amostras por segundo. O analisador é composto de três partes: uma unidade de rádio frequência $(\mathrm{RF})$, uma unidade de processamento do sinal e uma unidade remota de controle. A unidade de RF consiste de 16 transceptores, um para cada canal definido na norma IEEE 802.15.4 na faixa de frequência de $2,4 \mathrm{GHz}$. Os transceptores fornecem os pacotes recebidos e o valor do nível do sinal recebido (RSSI). A unidade de processamento do sinal adiciona uma marca temporal e armazena os dados em uma memória FIFO. Os dados são enviados via Ethernet para a unidade de controle remoto, que é uma aplicação executando em um computador, a qual processa e mostra os dados online ou 
offline ao usuário. A proposta não trata os dados capturados pelo sniffer, ou seja, não faz a decriptação, organização e filtragem dos dados.

Da mesma forma que as outras ferramentas baseadas em sniffer apresentadas anteriormente, esta também faz uso de um computador ligado ao sniffer para captura dos dados, o que, na maioria das vezes, inviabiliza seu uso em campo, ficando restrito ao uso em laboratórios.

\section{Dispositivo de campo WH com analisador de rede integrado}

Lorençato (2013) propôs um analisador de rede integrado ao dispositivo de campo WH. O sistema proposto realiza a leitura simultânea em todos os canais do espectro de $2,4 \mathrm{GHz}$ definidos na norma IEEE 802.15.4 e armazena as informações em uma memória não volátil (cartão de memória) no próprio dispositivo de campo. O sistema ainda possui um módulo GPS integrado para sincronizar as aquisições e posicioná-las geograficamente quando utilizado em campo aberto. A estrutura de RF é composta por 17 rádios, sendo que 16 são utilizados como rádios de aquisição, cada um sintonizado em um canal específico. Cada rádio é composto por um microcontrolador onde parte da pilha WH responsável pela recepção dos dados é implementada. Com isso, pode-se fazer uma interpretação local dos dados, descartando bytes de sincronismo, quadros mal formatados, inválidos ou com erros de validação, reduzindo o fluxo das informações capturadas, armazenadas, transferidas e analisadas pela unidade de análise dos dados. $\mathrm{O}$ décimo sétimo rádio está integrado à unidade de coleta dos dados e tem a habilidade de se unir à rede, rotear pacotes e responder a comandos. Assim, o analisador de rede passa a ser também um dispositivo de campo WH. Este sistema apresenta a vantagem de armazenar os dados localmente, ou seja, não é necessária a conexão do sniffer com um computador, possibilitando seu uso em ambientes reais. A ferramenta também possibilita a leitura dos dados coletados em tempo real, através da própria rede $\mathrm{WH}$.

\section{Proposta de uma nova ferramenta para monito- ramento de redes WH}

Na seção anterior, foram apresentadas as ferramentas para inspeção e monitoramento de redes WH existentes atualmente na literatura. Como se pode observar, ainda não existe uma ferramenta ótima e, por isso, novos trabalhos continuam sendo desenvolvidos, dada a importância de se ter uma ferramenta que permita analisar o comportamento dinâmico de uma rede $\mathrm{WH}$ em um ambiente real.

Este trabalho propõe uma nova abordagem para a inspeção de redes WH, unindo algumas das características presentes nas ferramentas já apresentadas, tais como: utilização de um cartão de memória para armazenar os dados coletados, evitando a necessidade de um computador próximo ao ponto de coleta de dados, e comunicação com o gateway através do protocolo HART-sobre-UDP para obtenção de dados da rede em tempo real.

A Figura 2 ilustra de maneira simplificada uma rede $\mathrm{WH}$ e a ferramenta proposta para análise da rede.

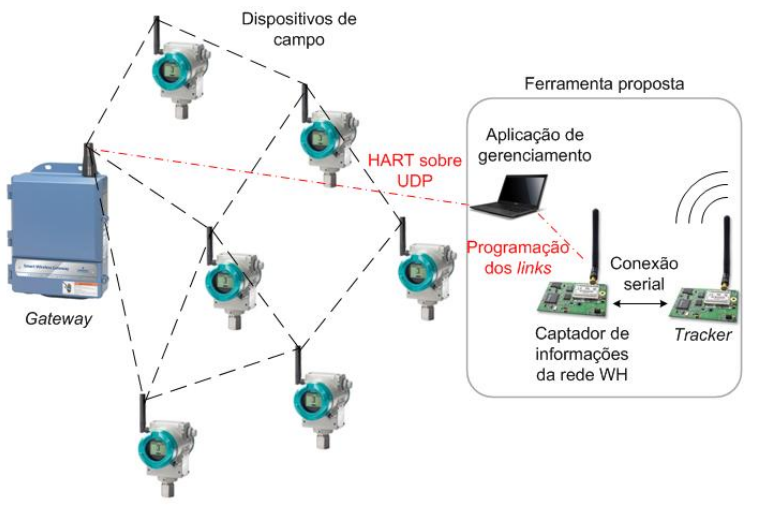

Figura 2. Ferramenta proposta para análise de redes WH

São necessários dois rádios IEEE 802.15.4, sendo um deles denominado de "captador de informações" e o outro, de "tracker". O rádio captador de informações é programado através de uma aplicação de gerenciamento, utilizando o protocolo HART-sobreUDP. Considerando que a rede já está estabelecida, ou seja, todos os dispositivos de campo já estão agregados, a aplicação de gerenciamento solicita informações ao gateway com o propósito de obter um mapeamento da rede. Inicialmente, solicita-se ao gateway a lista de todos os dispositivos que já se uniram à rede. Com os dados obtidos, a aplicação efetua a programação dos links dos vizinhos a serem inspecionados. Dessa forma, o rádio captador de informações obtém as comunicações realizadas entre os nós e o reconhecimento de mensagens (ACK). Assim, tem-se um sniffer capaz de detectar os pacotes em todos os canais WH, não sendo necessária a utilização de 15 rádios. Utiliza-se apenas um rádio que é programado dinamicamente conforme informações do escalonamento da rede. A inicialização da rede também pode ser capturada, através do armazenamento do processo de agregação dos dispositivos. Além do captador de informações, a ferramenta proposta utiliza um segundo rádio, denominado tracker. O tracker é conectado ao captador através de uma porta serial e tem duas funções principais: armazenar os dados coletados pelo captador em um cartão de memória e realizar a medição de energia no canal que está sendo utilizado em um dado instante de tempo. À medida que o captador de informações vai programando seu rádio para receber os pacotes que estão trafegando em todos os canais da rede $\mathrm{WH}$, os dados que estão sendo obtidos são enviados para sua porta serial, que por sua vez está conectada à porta serial do tracker, que faz a leitura 
dos dados e armazena-os em um cartão de memória para posterior análise. O tracker também tem a importante função de fazer a medição de energia no canal que está sendo utilizado. Quando o captador inicia o envio ou recebimento de uma mensagem, ele informa ao tracker qual o canal a ser utilizado, e este faz a medição do nível de energia durante a comunicação dos dados. Com isso, podem-se obter informações a respeito de interferências na rede, correlacionando o nível de energia medido com a recepção ou não de um pacote. Aliado à percepção da coexistência, estas informações são úteis para um futuro estudo sobre troca adaptativa de canais em redes WH.

A ferramenta ainda está em fase de desenvolvimento. O captador de informações e o tracker já foram testados separadamente e apresentam as funcionalidades descritas anteriormente. Os rádios utilizados foram desenvolvidos em (Muller, 2010). A ferramenta apresenta a vantagem de não necessitar um computador conectado ao sniffer e de não necessitar 15 rádios para captação em todos os canais. Apenas um rádio é necessário, devido à programação dinâmica que é feita no mesmo, fazendo com que seja sintonizado no instante e canal corretos.

\section{Resultados}

Este trabalho teve como propósito uma análise do estado da arte no que diz respeito a ferramentas para inspeção e monitoramento de redes WH. Além disso, propôs uma nova ferramenta que apresenta vantagens sobre as demais, dada a simplicidade de hardware envolvida e as informações que podem ser obtidas pela sua utilização. A Tabela 1 apresenta uma classificação das ferramentas apresentadas neste trabalho.

Tabela 1. Classificação das ferramentas de inspeção de redes WH.

\begin{tabular}{|c|c|c|c|c|}
\hline & \multicolumn{4}{|c|}{ Classificação quanto à: } \\
\hline Ferramenta & $\begin{array}{c}\text { Intrusão } \\
\text { na rede }\end{array}$ & $\begin{array}{c}\text { Aquisição } \\
\text { dos dados }\end{array}$ & Mobilidade & Tipo de análise \\
\hline $\begin{array}{c}\text { Porta de } \\
\text { manutenção }\end{array}$ & Passivo & $\begin{array}{c}\text { Requisição } \\
\text { /resposta }\end{array}$ & Conexão com PC & Online/Offline \\
\hline $\begin{array}{c}\text { HART-sobre- } \\
\text { UDP }\end{array}$ & Ativo & $\begin{array}{c}\text { Requisição } \\
\text { /resposta }\end{array}$ & Conexão com PC & Online/Offline \\
\hline $\begin{array}{c}\text { Wi-Analys } \\
\text { Passivo }\end{array}$ & $\begin{array}{c}\text { Captura } \\
\text { total }\end{array}$ & Conexão com PC & Online/Offline \\
\hline $\begin{array}{c}\text { Analisador } \\
\text { distribuído }\end{array}$ & Passivo & $\begin{array}{c}\text { Captura } \\
\text { total }\end{array}$ & Conexão com PC & Online/Offline \\
\hline $\begin{array}{c}\text { Analisador de } \\
16 \text { canais }\end{array}$ & Passivo & $\begin{array}{c}\text { Captura } \\
\text { total }\end{array}$ & Conexão com PC & Online/Offline \\
\hline $\begin{array}{c}\text { Dispositivo } \\
\text { WH com } \\
\text { analisador } \\
\text { integrado }\end{array}$ & Passivo & $\begin{array}{c}\text { Captura } \\
\text { total }\end{array}$ & Cartão de \\
memória & Offline \\
\hline $\begin{array}{c}\text { Captador + } \\
\text { tracker }\end{array}$ & $\begin{array}{c}\text { Ativo / } \\
\text { passivo }\end{array}$ & $\begin{array}{c}\text { Captura } \\
\text { total }\end{array}$ & $\begin{array}{c}\text { Cartão de } \\
\text { memória }\end{array}$ & Online/Offline \\
\hline
\end{tabular}

A ferramenta proposta é classificada como uma inspeção ativa e passiva, pois a aplicação de gerenciamento gera uma interferência ativa na rede. Esta aplicação é executada somente no início do processo de análise da rede e tem como objetivo obter o ma- peamento da rede. Após esta etapa inicial, tem-se uma inspeção passiva, ou seja, o captador e tracker obtêm os dados da rede sem interferir na mesma.

Com relação à aquisição dos dados, a captura total é mais eficiente do que a aquisição por meio de requisição/resposta, pois todos os dados que estão trafegando pela rede são capturados, enquanto a requisição/resposta depende de solicitações feitas pelo usuário ou aplicação de análise. No entanto, a captura total requer um processamento dos dados obtidos de modo a filtrar e selecionar as informações úteis.

Outra vantagem da ferramenta proposta diz respeito à mobilidade, pois não é necessário existir a conexão com um computador no ambiente de coleta dos dados, já que os dados são salvos em um cartão de memória para posterior análise. Este fato é bastante importante, pois o ponto de coleta dos dados pode ser de difícil acesso. A ferramenta proposta permite a análise offline dos dados, pela obtenção dos dados armazenados no cartão de memória e online, pelo envio e resposta de comandos especiais ao dispositivo coletor.

\section{Conclusão}

O protocolo WH está sendo cada vez mais aplicado nas indústrias devido ao fato de ser o primeiro padrão aberto para comunicação sem fio especialmente projetado para controle e monitoramento de processos em tempo real. Deste modo, torna-se necessário o desenvolvimento e uso de ferramentas para inspeção e análise das redes WH. Este trabalho apresentou uma análise das ferramentas já propostas na literatura, citando suas vantagens e desvantagens e também sua aplicabilidade em ambientes reais. Como ainda não existe uma ferramenta que contemple todas as características desejáveis para inspeção de redes $\mathrm{WH}$, esta área ainda é promissora e por isso merece estudo e novas pesquisas. Devido a isto, foi proposta uma nova ferramenta, que apresenta vantagens sobre as demais, especialmente no que diz respeito à mobilidade e simplicidade de construção. A ferramenta ainda está em desenvolvimento, e as próximas etapas incluem testes mais completos da integração entre o tracker e o captador de informações, além do desenvolvimento de uma aplicação que realiza a análise dos dados salvos no cartão de memória.

\section{Agradecimentos}

Agradecemos ao CNPq e a CAPES pela provisão de bolsas de estudo, sem as quais não seria possível a realização deste trabalho. Somos também gratos à Finep, financiadora do projeto E3 SA-WH. 


\section{Referências Bibliográficas}

Depari, A.; Ferrari, P.; Flammini A.; Lancellotti M.; Marioli, D.; Rinaldi S. and Sisinni E. (2009). Design and Performance Evaluation of a distributed WirelessHART Sniffer Based on IEEE1588. International IEEE Symposium on Precision Clock Synchronization for Measurement, Control and Communication. ISPCS 2009, Itália, pp. 1-6.

Han, S.; Song, J.; Zhu, X.; Mok, A. K.; Chen, D.; Nixon, M.; Pratt, W. and Gondhalekar, V. (2009). Wi-HTest: Compliance Test Suite for Diagnosing Devices in Real-Time WirelessHART Network. $15^{\text {th }}$ IEEE Real-Time and Embedded Technology and Applications Symposium, RTAS, pp. 327-336.

HART Communication Foundation - HCF (2009). Network Management Specification. HCF_SPEC-085, rev. 1.2.

Krätzig, M.; Rauchhaupt, L.; Schimschar, A. and Trikaliotis S. (2009). 16-Channel-Analyser for Parallel IEEE 802.15.4 Monitoring. IEEE Conference on Emerging Technologies \& Factory Automation, ETFA, 2009, pp. 1-4.

Lima, C. P.; Winter, J. M.; Muller, I.; Pereira, C. E. and Netto, J. C. (2012). Porta de Manutenção para Comissionamento e Análise Local de Redes WirelessHART. Congresso Brasileiro de Automática CBA 2012, Campina Grande. Anais do XIX Congresso Brasileiro de Automática, CBA 2012, vol. 1, pp. 4923-4929.

Lorençato A.; Muller, I.; Winter, J.; Kunzel, G.; Pereira, C. E. and Netto, J.C. (2013). WirelessHART Field Device with Integrated Network Analyser. Navcomp - Workshop em Sistemas Embarcados (WES), 2013, Rio Grande.

Muller I.; Pereira, C. E.; Netto, J. C.; Fabris, E. E. and Allgayer, R. (2010). Development of a WirelessHART Compatible Field Device. IEEE Instrumentation and Measurement Technology Conference, I2MTC, 2010, pp. 1430-1434.

Muller I.; Pereira, C. E.; Netto (2011). WirelessHART Field Devices. IEEE Instrumentation and Measurement Magazine, v.14, 2011, pp. 20-25. DOI: 10.1109/MIM.2011.6086896

Winter, J. M; Lima, C. P.; Muller, I.; Pereira, C.E. and Neto, J.C. (2011). WirelessHART Routing Analysis Software. I Simpósio Brasileiro de Engenharia de Sistemas Computacionais, SBESC, 2011, Florianópolis. Vol. 1, pp. 206-211. 